\title{
Charcot Spinal Arthropathy without Any Risk Factors: A Case Report
} \author{
of Korea \\ Corresponding author: Woo Jin Choe \\ Department of Neurosurgery, Konkuk \\ University Medical Center, Konkuk University \\ College of Medicine, 120-1, Neungdong-ro, \\ Gwangjin-gu, Seoul 05030, Republic of Korea \\ Tel: +82-2-2030-7357 \\ Fax: +82-2-2030-7359 \\ E-mail: s1nsman@kuh.ac.kr
}

Jihyun Oh, Soo Yeon Kim, Woo Jin Choe

Department of Neurosurgery, Konkuk University Medical Center, Konkuk University School of Medicine, Seoul, Republic

Received: August 26, 2020

Revised: September 12, 2020

Accepted: September 13, 2020

\begin{abstract}
Charcot spinal arthropathy (CSA) is a rare progressive disorder that occurs after the loss of neuroprotective sensation and proprioceptive perception. Although its etiology is unclear, studies have suggested that it is primarily caused by spinal cord injuries, and diabetes mellitus, syringomyelia, tertiary syphilis, and Parkinson's disease. Here, we describe a case of a 71-year-old woman with CSA without any risk factors. We report that CSA could occur without any prominent presentations or risk factors and may initially be misdiagnosed as pyogenic spondylitis.
\end{abstract}

Key Words: Arthropathy, neurogenic; Joint diseases; Spinal diseases; Spondylitis

\section{INTRODUCTION}

Charcot spinal arthropathy (CSA), also called "Charcot spine", is an uncommon condition that presents with destructive and degenerative joint processes ${ }^{6,8,10,11,15)}$. It usually occurs when afferent innervation is complicated ${ }^{11)}$, especially when nerves associated with pain sensation are damaged.

Historically, CSA was commonly thought to be related to tabes dorsalis secondary to syphilis ${ }^{11,15)}$. With the development of antibiotics, the incidence of CSA caused by syphilis has gradually reduced ${ }^{12,15)}$. Instead, CSA cases were found as a complication in patients with diabetes mellitus ${ }^{17)}$, spinal cord injury ${ }^{13,15)}$, Parkinson's disease, syringomyelia ${ }^{5}$, and congenital insensitivity to pain ${ }^{14)}$.

A clinical diagnosis of CSA is difficult because of the various or diverse times of onset of symptoms and the non-specific nature of its clinical signs ${ }^{1)}$. A diagnosis is usually made at an advanced stage of lesion formation with progressed bone destruction ${ }^{12)}$. Radiologic features of CSA include extensive bony destruction and regeneration, and it is sometimes difficult to differentiate these features from spondylitis or tumorous lesion in the vertebral columns ${ }^{6,10)}$. A biopsy can be performed for the suspected lesion if a differential diagnosis by imaging modalities alone is difficult. Characteristics of $\mathrm{CSA}^{11)}$ are described in Table 1.

Herein, we report a case of a patient with CSA who presented without any risk factors and was initially suspected to have tuberculous spondylitis.

\section{CASE REPORT}

A 71-year-old woman was referred to our hospital with a 1-year history of low back pain and pain in both legs. She had no history of spinal cord injury, neurological disease, or diabetes mellitus. She had visited a local hospital complaining of back pain and claudication that prohibited her from walking for more than $10 \mathrm{~min}$. Despite several nerve block procedures, the pain progressed. Magnetic resonance imaging (MRI) performed at another hospital showed irregular destruction of the L5-S1 vertebral endplates with rim enhancement around the L5-S1 disc space. Furthermore a fuzzy and extensive enhancement in the paravertebral structures was noted from L4-S1 (Fig. 1). She underwent a biopsy with laminectomy and dis-
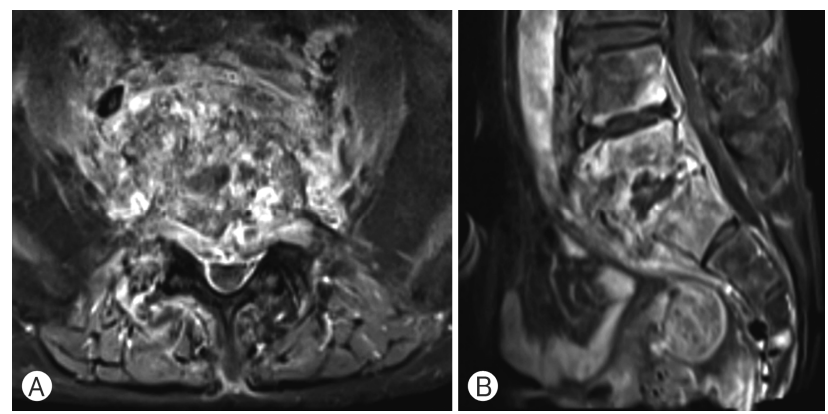

Fig. 1. Lumbar magnetic resonance images show irregular destruction of endplate of L5-S1 with rim enhancement around L5-S1 disc space. A fuzzy and extensive enhancement in the paravertebral structures was noted at the L4-5-S1 level. (A) Axial and (B) sagittal images. 
Table 1. Clinical characteristics of Charcot spinal arthropathy

\begin{tabular}{ll}
\hline \hline Characteristics & \multicolumn{1}{c}{ Description } \\
\hline Pre-existing conditions & Spinal cord injury \\
& Diabetes mellitus \\
& Syringomyelia \\
& Meningocele, myelomeningocele \\
& Peripheral neuropathies \\
& Congenital analgesia \\
& Parkinson's disease \\
& Transverse myelitis \\
Relevant spine levels & Thoracolumbar spine is most commonly \\
& affected region \\
Clinical symptoms & Back/Leg pain \\
& Presence of an audible cracking sound \\
& Lower extremity weakness \\
& Deformity \\
& Spasticity \\
& Hyperreflexia \\
Bladder/Anal dysfunction \\
Bone erosion of vertebral body \\
Osteophytosis \\
Stenosis of the intervertebral space \\
Presence of paravertebral masses \\
Facet destruction \\
Spondylodiscitis \\
Pyogenic spondylitis \\
Osteomyelitis \\
Paget's disease \\
local procedure, immobilization \\
Conservatived approach
\end{tabular}

cectomy from L4-S1 at another hospital upon suspicion of infective spondylitis. 'Cefazedone' was administered to her as an empirical antibiotic after biopsy. However, according to the medical record, there was no evidence of infection in the bones, tissues, or discs. The results from the biopsy and culture tests were negative for infection.

The back pain and radiculopathy worsened despite treatment, and she was transferred to our hospital for further evaluation. At presentation, she was afebrile and her laboratory tests showed no evidence of infection (erythrocyte sedimentation rate [ESR], $36 \mathrm{~mm} / \mathrm{hr}$, C-reactive protein [CRP], $0.11 \mathrm{mg} /$ dL). Computed tomography (CT) and MRI showed further destruction and enhancement around the L5-S1 disc spaces and signal change at the L4-5 level (Fig. 2). The images in Fig. 2 were obtained 2 months after the images in Figure 1. The radiculopathy was thought to be from bilateral L5-S1 foraminal stenosis secondary to bony destruction. Dual energy X-ray absorptiometry to measure bone mineral density revealed a T-score of -2.7 standard deviation in her right femoral neck. A whole-body bone scan showed no other findings besides active tracer uptake at the L4-5 level, which was consistent with arthritic changes. To exclude infection, biopsies were performed at the L4-5 level. The cortical bones were extremely hard and abnormally sclerotic, which made it difficult to insert and remove the biopsy needles. The pathological study showed non-neoplastic or non-infectious bony tissue with normal hematopoiesis, and the bony tissue showed probable ischemic damage with scant marrow cells (Fig. 3). The laboratory test results, including polymerase chain reaction for tuberculosis, gave no evidence of infectious diseases. Moreover, all results of Gram staining, fungal staining, and bacterial cultures were negative for infection. As there was no evidence of infection, antibiotics were not administered.

To relieve symptoms, the patient underwent a posterior instrumentation with fusion using a cage at the L4-5-S1 level and bilateral L5-S1 foraminal decompression (Fig. 4). At the time of the operation, extensive granulation and adhesive tissues were observed and removed. We performed the biopsy
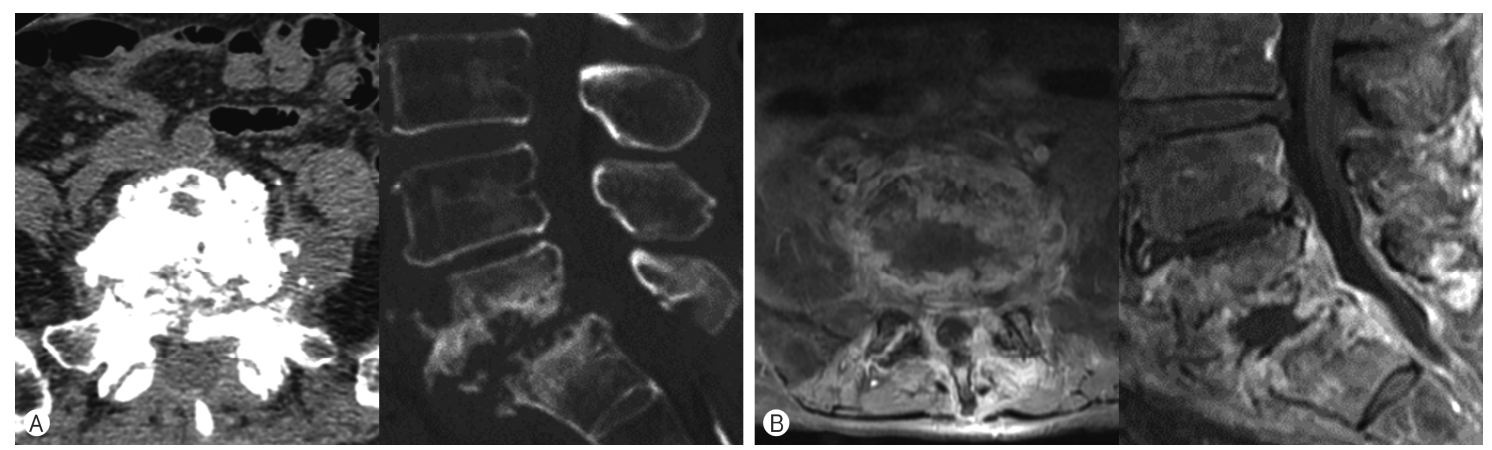

Fig. 2. (A) Lumbar computed tomography scan and (B) magnetic resonance images (MRI) showing extensive bony destruction and enhancement around the disc space at the L4-5-S1 disc level. The findings mimicked pyogenic or tuberculous spondylitis. (B) These MRI images were obtained 2 months after the images in Figure 1. 
and culture test of obtained tissue samples. However, the results showed no evidence of infection, including tuberculosis. Pathological study of the bone biopsy revealed fibrocartilage tissue without inflammation. The patient's back pain and radiculopathy improved after the surgery. A follow-up imaging study obtained 10 months postoperatively showed continued progression of abnormal bony destruction and regeneration even after fixation and fusion (Fig. 5)

After thorough consideration of the clinical and pathological findings, the diagnosis of CSA was suggested.

\section{DISCUSSION}

CSA is a progressive and destructive arthropathy that involves the vertebral bodies, discs, and articular facets ${ }^{1,15)}$.

In 1868, Charcot $^{4)}$ first described CSA, and in 1884, Kronig ${ }^{9)}$ reported the first case of CSA in patients with tabes dorsalis ${ }^{2,11,12)}$. In the past, the primary possible cause of CSA had been syphilitic tabes dorsalis. However, with the reduction in the number of patients with syphilis, the number of patients with CSA secondary to tabes dorsalis has also decreased. Recently, other causes, including traumatic spinal cord injury, diabetic mellitus,

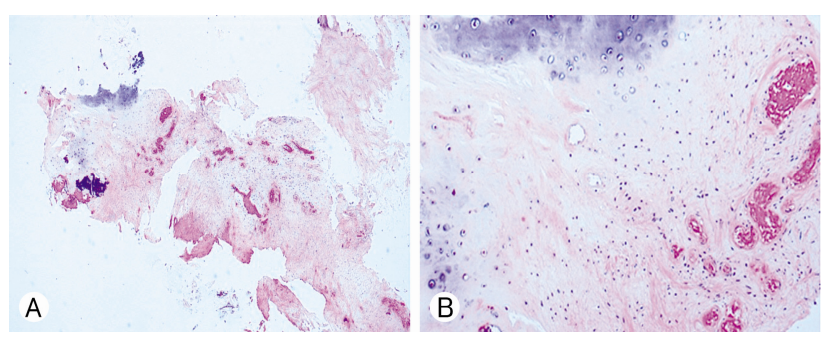

Fig. 3. Histological findings of bone biopsy specimen. (A) Hematoxylin and eosin stain (H\&E), $\times 100$ magnification and (B) H\&E, $\times 200$ magnification. The major findings were chronic inflammation and ischemic damage with reactive bone formation. No evidence of infection or neoplasm was found.
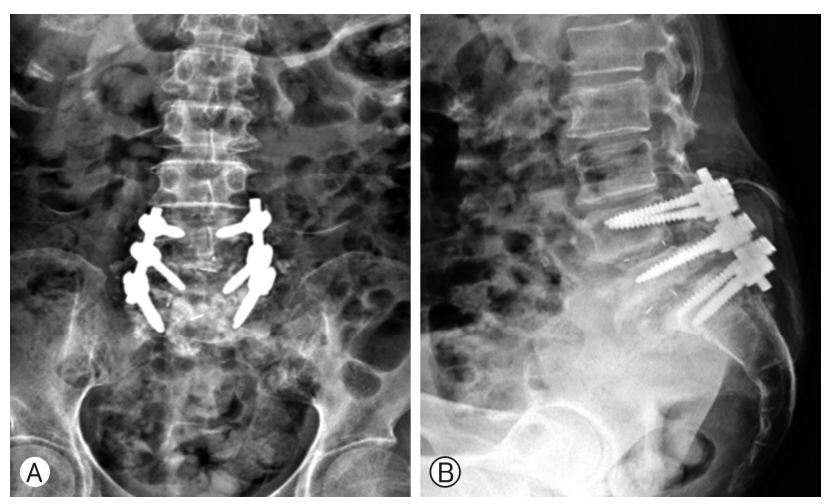

Fig. 4. Posterior instrumentation with fusion using a cage were performed at the L4-5-S1 level and bilateral L5-S1 foraminal decompression. (A) Anteroposterior and (B) lateral images. syringomyelia, congenital pain insensitivity, and peripheral nerve injury have been identified as predisposing factors for $\mathrm{CSA}^{8,11,15,16)}$

The pathogenetic mechanism of CSA has not been clearly understood. Decreased proprioception and pain sensation are widely accepted predisposing factors for CSA, which may cause abnormal movement of the joints. Because of the loss of deep pain sensation, most patients lack symptoms in the early stages of the disease, and they come to the hospital only after considerable progression of the disease ${ }^{12)}$.

Repetitive minor stress from the abnormal joint movements causes damage to the bones, cartilage, and joints. Gradually, cartilage destruction occurs, and the joint space narrows, leading to edema and effusion at the joint. At this point, both hypertrophic and atrophic changes can occur. Hypertrophic changes cause sclerosis of bones, osteophytes, and pseudoarthrosis formation, while atrophic changes cause osteolysis and destruction of bones. If the joint is not stabilized and is stressed repetitively, this process gradually progresses ${ }^{15)}$. In the current case, the patient worked very hard in restaurants, repeating a motion of bending and stretching the waist. CSA was thought to be caused by repetitive microtrauma to her lumbar spine.

It is hard to diagnose CSA early because of its non-specific symptoms, and patients usually complain of spinal deformity, back pain, and the presence of an audible cracking sound. For this reason, CSA is commonly diagnosed in a progressed state with massive bony destruction. Early suspicion of CSA is important in preventing further progression of bony destruction.

Radiographically, CSA presents with disc space narrowing, vertebral body erosion, osteosclerosis, and facet joint destruction $^{6,11,15)}$. The thoracolumbar spine is often the most commonly affected ${ }^{11)}$. Damage to all 3 vertebral columns induces instability leading to spinal deformity, including kyphosis and scoliosis $^{2)}$. Occasionally, findings such as paraspinal soft tissue mass or abnormal fluid are seen. Peripheral discs are enhanced
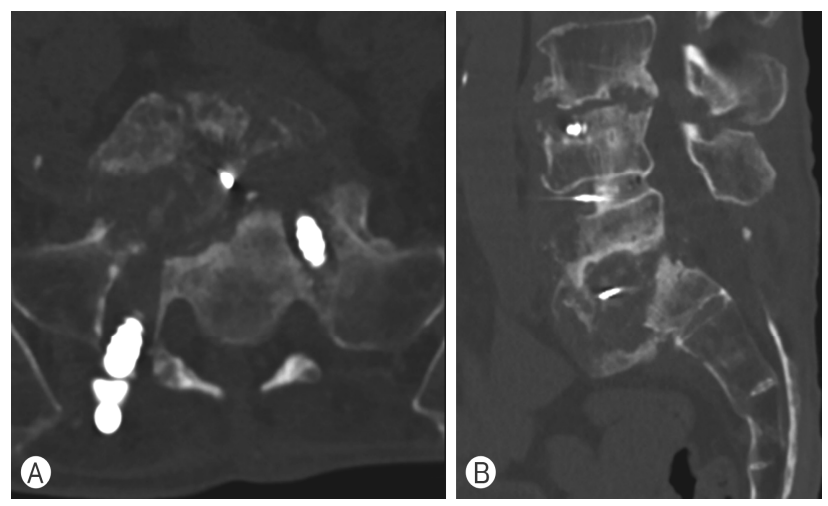

Fig. 5. Follow-up lumbar computed tomography scan obtained 10 months postoperatively showed abnormal bony resorption around the pedicle screws. (A) Axial and (B) sagittal images. 
on imaging with contrast agents. CT is useful in assessing bone destruction and osteophyte formation, while MRI is valuable in the evaluation of the adjacent soft tissue ${ }^{11)}$.

A primary differential diagnosis should be made with spondylodiscitis, and other considerations include severe degenerative disc disease and metastatic disease, both of which can show similar imaging findings ${ }^{15}$. The clinical course is important in diagnosis, but pathologic confirmation by biopsy may be necessary. Histologic findings are usually non-specific findings and chronic inflammation ${ }^{2}$. After excluding other suspected diseases using imaging, laboratory, and pathological tests, the diagnosis of CSA can be made. In the present case, pyogenic spondylitis was initially suspected based on imaging tests. In this patient, ESR and CRP, which are sensitive laboratory indicators of pyogenic spondylitis, were within normal range or slightly elevated. There was no evidence of infection in microbial culture tests from the biopsy. In addition, the patient had no notable medical history, such as spinal cord injury, diabetic mellitus, or syringomyelia. We were able to diagnose this case as CSA by considering all clinical, laboratory, and pathological findings, even though the patient had no known risk factors.

Cases of CSA associated with various predisposing factors have been reported ${ }^{13,14,17)}$. According to our literature search, cases of CSA without any risk factors are rarely reported. Rare case of idiopathic Charcot arthropathy of the elbow and knee have been reported in the literature ${ }^{3,7)}$. This is the first reported case of Charcot arthropathy of the spine without any risk factors. In the case of idiopathic Charcot arthropathy without other risk factors, diagnosis is possible only after excluding other predisposing factors. It is also important to check the patient's occupation or activities to exclude the possibility of microtrauma caused by exercise or repetitive motion.

There is no definite or universally accepted treatment for CSA. Management options include conservative and surgical treatments $^{8}$. Immobilization of the affected joint and weightbearing restrictions are recommended to treat CSA. Conservative treatment may be considered for patients without neurologic deficit or severe symptoms. If conservative treatment fails or instability persists, surgical treatment is required ${ }^{17)}$. Surgical treatment consists of spinal fusion and instrumentation with extensive lesion debridement for decompression ${ }^{8}$. The patient in this case received surgical treatment because her pain was not under control and bony destruction progressed despite conservative treatment. The pain caused by spinal stenosis was relieved after foraminal decompression and the joint was stabilized through posterior instrumentation and fusion. Reports describing the outcomes or complications after surgical treatment of CSA are rare ${ }^{8)}$. Some authors have reported adverse outcomes or complications that require reoperation, such as hardware failure, deep infections or the development of additional Charcot lesions ${ }^{6,11)}$. In this case, abnormal active degeneration was noted, especially in the bone around the pedicle screw. Long-term follow-up is necessary owing to the nature of the disease process and the potential for progressive instability.

\section{CONCLUSION}

CSA shows a destruction of affected spinal regions and usually coincides with diabetes or spinal cord injury. We describe a case of CSA without any risk factors. In case of progressive spinal destruction, CSA may be considered as a differential diagnosis despite the absence of known risk factors.

\section{CONFLICTS OF INTEREST}

No potential conflict of interest relevant to this article was reported.

\section{REFERENCES}

1. Aebli N, Pötzel T, Krebs J: Characteristics and surgical management of neuropathic (Charcot) spinal arthropathy after spinal cord injury. Spine J 14:884-891, 2014

2. Barrey C, Massourides H, Cotton F, Perrin G, Rode G: Charcot spine: Two new case reports and a systematic review of 109 clinical cases from the literature. Ann Phys Rehabil Med 53:200220, 2010

3. Blanford AT, Keane SP, McCarty DJ, Albers JW: Idiopathic Charcot joint of the elbow. Arthritis Rheum 21:723-726, 1978

4. Charcot JM: Sur quelques arthropathies qui paraissent dépendre d'une lésion du cerveau ou de la moelle épinière. Arch Phys Norm Pathol 1:161-178, 1868

5. Deng X, Wu L, Yang C, Xu Y: Neuropathic arthropathy caused by syringomyelia. J Neurosurg Spine 18:303-309, 2013

6. Goetz LL, McAvoy S, Zakrzewski K: Neuropathic spinal arthropathy leading to spine disruption, spinal cord transection, and aortic displacement: Brief case report. Spinal Cord Ser Cases 4:91, 2018

7. Hanson LF, Hanson CG, Barner KL: Idiopathic Charcot arthropathy of the knee presenting as a bicondylar tibial plateau fracture. J Am Acad Orthop Surg Glob Res Rev 2:e032, 2018

8. Hong J, Sanfilippo JA, Rihn J, Fernandez C, Winegar CD, Friel $\mathrm{B}$, et al.: Complications in the management of Charcot spinal arthropathy. J Neurosurg Spine 11:365-368, 2009

9. Kronig G: Spondylolisthese bei einem Tabiker. Z Klin Med 7: S165, 1884

10. Ledbetter LN, Salzman KL, Sanders RK, Shah LM: Spinal neuroarthropathy: Pathophysiology, clinical and imaging features, and differential diagnosis. Radiographics 36:783-799, 2016

11. Lee D, Dahdaleh NS: Charcot spinal arthropathy. J Craniovertebr Junction Spine 9:9-19, 2018

12. Moreau S, Lonjon G, Jameson R, Judet T, Garreau de Loubresse C: Do all charcot spine require surgery? Orthop Traumatol Surg Res 100:779-784, 2014

13. Morita M, Miyauchi A, Okuda S, Oda T, Yamamoto T, Iwasaki 
M: Charcot spinal disease after spinal cord injury. J Neurosurg Spine 9:419-426, 2008

14. Sliwa JA, Rippe D, Do V: Charcot spine in a person with congenital insensitivity to pain with anhydrosis: A case report of re-diagnosis. Arch Phys Med Rehabil 89:568-571, 2008

15. Staloch MA, Hatem SF: Charcot spine. Emerg Radiol 14:265269, 2007
16. Suda Y, Shioda M, Kohno H, Machida M, Yamagishi M: Surgical treatment of Charcot spine. J Spinal Disord Tech 20:8588, 2007

17. van Eeckhoudt S, Minet M, Lecouvet F, Galant C, Banse X, Lambert M, et al.: Charcot spinal arthropathy in a diabetic patient. Acta Clin Belg 69:296-298, 2014 